\title{
Effects of feeding practices on the diversity of faecal short chain fatty acids in 1-4 months infants in Pakistani cohort
}

\author{
Mehwish Durrani ${ }^{1}$, Ashfaq Ahmad Shah Bukhari ${ }^{2}$, Muhammad Abubakr ${ }^{3}$, Kalsoom Tariq ${ }^{4}$, Aziz Ur Rehman ${ }^{5}$, Muhammad Shafiq $^{6}$
}

\section{Submitted:}

September 9, 2020

Accepted:

November 4, 2020

\section{Author Information}

${ }^{1,5,6}$ Biochemistry Department

Rehman Medical College, Peshawar Pakistan

${ }^{2}$ Physiology Department

Rehman Medical College, Peshawar Pakistan

${ }^{3}$ Pak International Medical College,

Peshawar Pakistan

${ }^{4}$ Biochemistry Department,

Khyber Girls Medical College, Peshawar Pakistan

\section{Corresponding Author}

Mehwish Durrani

Associate Professor

Rehman Medical College RMI Peshawar

Email: drmehwishdurrani@gmail.com

\begin{abstract}
Introduction: The most important product of fermentation of non-digestible carbohydrates is short chain fatty acids. Formation of these metabolites are mainly dependent on diet and gut microbial colonization. Literature has been supportive of the fact that short chain fatty acids play significant role in chronic inflammation (allergic diseases) and host physiology and metabolic processes including obesity and diabetes. Little is known about the differences in the metabolism between breast-fed and bottle-fed infants in our country. This study was designed to determine the differences in the metabolism between breast-fed and bottle-fed infants in Pakistani cohort.

Material \& Methods: A cross sectional study was carried out in 50 healthy infants. Enrollment of these children was done from different centers and localities in Peshawar. Stool samples were collected from the included infants and short chain fatty acids were studied by using Gas Chromatography.

Results: A total of 50 healthy infants ( 25 breast-fed and 25 bottle-fed) with mean age (mean \pm sd) 78.40 35.88 days (range: $1-4$ months) were included in this survey. Breast feeding was associated with lesser absolute concentrations of total short chain fatty acids. Among short chain fatty acids, acetates were more in breast feeding infants (median 651, $\mathrm{p}=0.001$ ) compared to formula feeding infants while propionates (median 81.06, $\mathrm{p}=0.02$ ), butyrates (median 31.86, $\mathrm{p}=0.007$ ) and iso-butyrates (median $3.76, \mathrm{p}=0.003$ ) were dominant in FFI which indicated an important role of short chain fatty acids in human health.

Conclusion: It may be concluded that feeding practices strongly influence the gut microbial metabolism and metabolites (short chain fatty acids).
\end{abstract}

Key Words: breast feeding infants, formula feeding infants, gut microbiota, short-chain fatty acids

The authors declared no conflict of interest and agreed to be accountable for all aspects of the work in ensuring that questions related to the accuracy or integrity of any part of the work are appropriately investigated and resolved. All authors contributed substantially to the planning of research, questionnaire design, data collection, data analysis and write-up of the article as part of a $\mathrm{PhD}$ student research team at Khyber Medical University, Peshawar.

This article may be cited as: Durrani, M., Bukhari, AAS., Abubakr, M., Tariq, K., Rehman, A., Shafiq, M. Effects of feeding practices on the diversity of faecal short chain fatty acids in 1-4 months infants in Pakistani cohort. Reh J Health Sci. 2020;2(2). 36-39

\section{INTRODUCTION}

When non-digestible carbohydrates namely dietary fibers undergo fermentation by large bowel bacteria, they produce Short Chain Fatty Acids (SCFAs). ${ }^{1}$ Researches have reported profound effects of dietary fibers on general health. This included a decrease in body weight and fat mass by increasing the post-meal satiety and decreases in the aggressiveness of diabetes. The effects are attributed to the fermentation of dietary fibers by large gut bacteria via producing various metabolic products like SCFAs, which influence the energy homeostasis through the host physiology. ${ }^{2}$ The three main SCFAs are $60 \%$ acetates (C2), $25 \%$ propionates (C 3 ) and $15 \%$ butyrates (C4). For colonic epithelium, butyrate is considered as a main energy entity which plays a significant role in intestinal level and extra-intestinal level. They regulate transepithelial fluid transport, alleviate oxidative status and mucosal inflammation thus preventing colorectal neoplasm and enhancing the epithelial defense barrier. Extra-intestinally they alleviate metabolic diseases including hemoglobinopathies, insulin resistance, ischemic stroke and hypercholesterolemia. ${ }^{3,4}$ SCFAs are used as a source of energy by the intestinal cells and by the intestinal micro flora. They influence and affect the intestinal environment and the colonic physiology and are involved in various mechanisms of host signaling. ${ }^{5}$

SCFAs formation by the intestinal bacteria are species-specific which is evident by the production of different acids produced in the gut microflora. The carbohydrates that resist digestion in small gut are fermented by the resident microbes present in the large gut. The gut microbiota of human comprises of 1013 - 1014 microbes amounting to $>1 \mathrm{~kg}$ of total body weight. The main resultant of bacterial fermentation of resistant carbohydrates are SCFAs. SCFAs provide a vast variety of health benefits. ${ }^{1}$ In normal healthy individual, daily production of SCFAs was measured to be 300 $\mathrm{mmol} /$ day and only $10 \mathrm{mmol} /$ day is excreted out. Thus the main bulk of it is being used by the colonic cells. ${ }^{6,7}$

Almost $60-70 \%$ of the total energy needed by the colonic cells is provided from the metabolization of SCFAs; butyrate being the main fuel for the colonocytes. ${ }^{7,8}$ After the production of SCFAs they are transported via the blood stream to different organs of the body. The SCFAs modify and regulate different system like endocrine, blood, gut and immune systems.

Gut microbiota that developed since the time of birth and the 
metabolites produced by the microbiota plays an important role in maintaining health of an infant throughout life. It is believed that the microbiota developed in early age imperative to normal health and physiology and is dependent upon the feeding practices. Breast feeding and formula feeding results in different composition and proportion of gut bacteria. ${ }^{9}$ The SCFAs concentrations are significantly different in both. The levels of SCFAs are higher in formula fed infants and are associated with the higher diversity of gut microbiome in comparison to breast fed infants. The increased variety in the gut microbiota leads to higher abilities to metabolize substrates in to SCFAs in the gastrointestinal tract. Whereas in breast fed infants both the bacterial diversity and metabolites levels are relatively low. ${ }^{10}$ The proteolytic products are found to be higher in formula fed infants and are attributed to low levels of carbohydrates available in the absence of human milk oligosaccharides. This leads a complete metabolism of proteins available for energy provision. Higher levels of SCFAs in formula fed infants may cause a quite few metabolic consequences. ${ }^{11}$ It has been reported that very low quantity of n-butyric acid was present in breast fed infants. ${ }^{12,13}$ In formula-fed infants, the levels of propionic acids are higher when compared with breast fed infants. In breast fed infants higher levels of lactates and acetates are found due to the presence of lactate producing bacteria, Bifidobacterium, Enterococci and Lactobacilli whereas n-butyric acid and propionic acid are found in formula fed infants with predominant form of Clostridia, Bacteroides and Fusobacterium. ${ }^{13}$

\section{MATERIALS \& METHODS}

The aim of this observational study was to determine the difference in the metabolism between breast fed and formula fed infants in Pakistani cohort. Based on median difference of 14.3 $\mathrm{mmol} / \mathrm{kg}$ total SCFAs between breast fed and formula fed infants at 2 months of age from a study carried out previously 13 and considering margin of error of 5\%, a total of 25 infants were required in each group (total $n=50$ ) for this trial. The study was explained to the mothers in local language and an informed consent was obtained from all the mothers who were willing to participate.

The study was approved by the Khyber Medical University Ethics board (KMU-Ethics Board) on 27th of October 2016 for a period of 4 years under the study reference number DIR/KMUEB/PZ/000316. This study was carried out in Peshawar, Khyber Pakhtunkhwa.

For the purposes of this study, breast feeding was defined by the WHO. According to WHO, breast feeding 'means that the infant's predominant source of nourishment has been breast milk'.

However, the infant may also have received liquids (water and water-based drinks, fruit juice) ritual fluids and ORS, drops or syrups (vitamins, minerals and medicines). Similarly, formula feeding infants means 'infant's predominant source of nourishment has been formula milk'. However, the infant may also have received liquids (water and water-based drinks, fruit juice) ritual fluids and ORS, drops or syrups.
Screening of the Participants

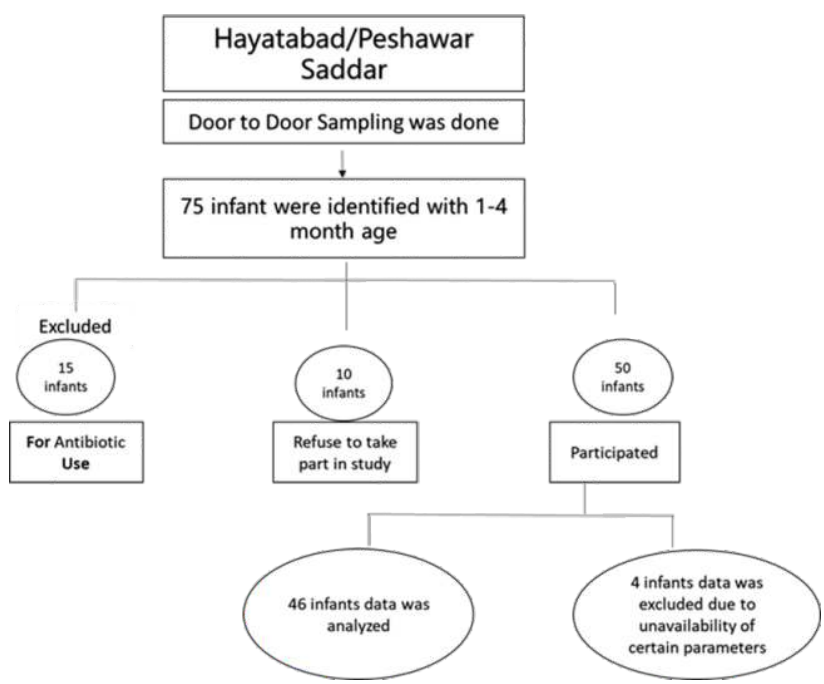

Figure 1: Flow chart of participant screening

\section{Laboratory Methods}

\section{Handling of stool sample and analysis time}

Collection kits for faeces were given to all mothers after they had signed the consents forms. Every kit had a fecal collection container with a plastic covering, an ice pack (to keep the sample cold) a spatula (to assist collecting the sample from the diaper in the pot) and a pair of gloves (for sample handling). The mothers were asked to collect the fecal sample on a tentatively agreed date and if not possible, then the sample was collected on a later agreed day. The researcher was informed immediately to pick the sample for further processing and analysis. All the stool samples were processed within $12 \mathrm{hrs}$ after receiving the sample from the mothers.

\section{Initial stool processing}

Every sample was received in anaerobic cold environments, weighed alongside with fecal collection pot and homogenized with clean spatula. The undigested food debris were removed (if any) during this process. Approximately $200 \mathrm{mg}$ of stool sample was weigh up in $2 \mathrm{ml}$ screw-cap micro tubes in triplicate and straightaway stored at $-80 \quad$ C. One gram of sample was stored in $5 \mathrm{ml}$ bijoux tubes adding 3 glass beads to each tube. To these tubes $1 \mathrm{ml}$ of $1 \mathrm{M} \mathrm{NaOH}(\mathrm{w} / \mathrm{v})$ was added to aid retaining the volatile SCFAs and was kept at -20 C until further analysis.

\section{SCFAs analysis}

SCFAs were studied via Gas chromatography Trace GC 2000 series (thermos Quest CE instruments, Manchester, UK). The SCFAs were first extracted by using diethyl ether then subjected to GC. The samples were sent to "The College of Medicine, Veterinary, and Life Sciences, University of Glasgow' for analysis. Diethyl ether extraction method was used via Gas chromatography for extraction of SCFAs. Diethyl ether which is an organic solvent is used widely. In a mixture of extract, diethyl ether form two phases after adding ortho-phosphoric acid (strong acid) that increases the dissociation constant (pKa value). A total of $100 \mathrm{mg}$ of the freezedried sample was taken in duplicate in $15 \mathrm{ml}$ corning tubes (polypropylene centrifuge tubes). To homogenize the sample, 300 $\mu \mathrm{l}$ of distilled water was added and vortexed. To this mixture, 100 


\section{Laboratory Methods}

\section{Handling of stool sample and analysis time}

Collection kits for faeces were given to all mothers after they had signed the consents forms. Every kit had a fecal collection container with a plastic covering, an ice pack (to keep the sample cold) a spatula (to assist collecting the sample from the diaper in the pot) and a pair of gloves (for sample handling). The mothers were asked to collect the fecal sample on a tentatively agreed date and if not possible, then the sample was collected on a later agreed day. The researcher was informed immediately to pick the sample for further processing and analysis. All the stool samples were processed within $12 \mathrm{hrs}$ after receiving the sample from the mothers.

\section{Initial stool processing}

Every sample was received in anaerobic cold environments, weighed alongside with fecal collection pot and homogenized with clean spatula. The undigested food debris were removed (if any) during this process. Approximately $200 \mathrm{mg}$ of stool sample was weigh up in $2 \mathrm{ml}$ screw-cap micro tubes in triplicate and straightaway stored at $-80 \quad$ C. One gram of sample was stored in $5 \mathrm{ml}$ bijoux tubes adding 3 glass beads to each tube. To these tubes $1 \mathrm{ml}$ of $1 \mathrm{M} \mathrm{NaOH}(\mathrm{w} / \mathrm{v})$ was added to aid retaining the volatile SCFAs and was kept at $-20 \quad \mathrm{C}$ until further analysis.

\section{SCFAs analysis}

SCFAs were studied via Gas chromatography Trace GC 2000 series (thermos Quest CE instruments, Manchester, UK). The SCFAs were first extracted by using diethyl ether then subjected to GC. The samples were sent to 'The College of Medicine, Veterinary, and Life Sciences, University of Glasgow' for analysis. Diethyl ether extraction method was used via Gas chromatography for extraction of SCFAs. Diethylether which is an organic solvent is RESULTS

A total of 50 infants were recruited for this trial. The mane age of these included infants was $78.40+35.88$ days (mean + sd). The demographic and anthropometric data consist of family size, number of siblings, age of infants at the time of assessment, SDS height, SDS weight and SDS BMI (Table1). Independent sample ttest was applied to calculate p-value.

Table 1: Anthropometric and demographic characteristics of infants

\begin{tabular}{llll}
\hline Variable & Mean & St Dev. & p-Value \\
\hline Height $(\mathrm{cm})$ & 53.42 & 5.84 & 0.94 \\
Weight $(\mathrm{kg})$ & 5.37 & 1.01 & 0.22 \\
SDS Height & -2.75 & 3.10 & 0.81 \\
SDS Weight & -0.53 & 2.05 & 0.44 \\
BMI (kg/m2) & 19.17 & 4.11 & 0.25 \\
SDS BMI & 1.59 & 2.30 & 0.29 \\
Age at Assessment (days) & 78.40 & 35.88 & 0.38 \\
Family Size (n) & 6.78 & 3.30 & 0.89 \\
No. of siblings (n) & 2.00 & 2.07 & 0.68 \\
\hline
\end{tabular}

The data was non-parametric, so descriptive statistics was expressed as median and interquartile range (Table 2)

Table 2: Descriptive statistic of infants gut

short chain fatty acids

\begin{tabular}{ccccc}
\hline \multicolumn{1}{c}{ Variables } & Minimum & Medium & Maximum & IQR \\
\hline Acetates C2 $(\mu \mathrm{mol} / \mathrm{gm})$ & 191.0 & 484.0 & 1012.0 & 292.0 \\
Propionates C3 $(\mu \mathrm{mol} / \mathrm{gm})$ & 15.64 & 58.54 & 278.00 & 43.21 \\
Isobutyrates C4 $(\mu \mathrm{mol} / \mathrm{gm}$ & 1.000 & 3.171 & 11.460 & 2.36 \\
Butyrates C4 $(\mu \mathrm{mol} / \mathrm{gm})$ & 3.83 & 27.32 & 109.00 & 55.24 \\
Isovalerate C5 $(\mu \mathrm{mol} / \mathrm{gm})$ & 1.34 & 5.79 & 34.57 & 5.58 \\
valerate C5 $(\mu \mathrm{mol} / \mathrm{gm})$ & 0.18 & 10.36 & 36.86 & 11.46
\end{tabular}

Mann Whitney U-test was applied to assess the comparison of gut microbial SCFAs between breast-fed and formula-fed infants (Table $3)$.

Table 3: Comparison of gut microbial SCFAs between breast-fed and formula-fed infants

\begin{tabular}{llll}
\hline Variable & Mean & St Dev. & p-Value \\
\hline Acetates C2 $(\mu \mathrm{mol} / \mathrm{gm})$ & 651 & 433 & $0.001 \dagger \dagger$ \\
Propionates C3 $(\mu \mathrm{mol} / \mathrm{gm})$ & 51.87 & 81.06 & $0.02 \dagger$ \\
Isobutyrates C4 $(\mu \mathrm{mol} / \mathrm{gm})$ & 2.74 & 3.76 & $0.003 \dagger \dagger$ \\
Butyrates C4 $(\mu \mathrm{mol} / \mathrm{gm})$ & 10.40 & 31.86 & $0.007 \dagger \dagger$ \\
Isovalerate C5 $(\mu \mathrm{mol} / \mathrm{gm})$ & 4.03 & 7.99 & 0.11 \\
valerate C5 $(\mu \mathrm{mol} / \mathrm{gm})$ & 8.98 & 12.18 & 0.07 \\
\hline
\end{tabular}

Significant $\mathrm{p}$ value $(\dagger \mathrm{P}<0.05, \uparrow \dagger \mathrm{P}=<0.01, \uparrow \dagger \dagger \mathrm{P}<0.0001)$

DISCUSSION

It is well supported by the literature that the fecal flora of breastfed and formula-fed infants are distinct from each other and have different electrolytes. Diet not only alters the composition of the metabolites formed by the gut microbiota but also determines the composition of colonic microbiota species. ${ }^{14}$ Anaerobes the acetate producers, propionates are largely produced by Phylum Bacteroidetes and some Firmicutes. Lactate utilizing species convert, lactate which is formed by many groups into acetates, butyrate and propionate. ${ }^{15}$ SCFAs are volatile acids formed in large gut by gut microbiota as products of fermentation from different food components which escape digestion in the small intestine. ${ }^{5}$ The infants gut is influenced mainly by predominance of whey or casein proteins, buffering capacity ${ }^{16}$, nucleotide content ${ }^{17}$ and lactoferrin and iron content. ${ }^{18}$ Physiological factors such transit time also play important role. In past dedicated researches were done on microbial population. It has been reported that the differences in fecal flora cannot be attributed to a single entity and may be related to dietary and differences in formula and human milk. ${ }^{19}$

In this study the levels of fecal SCFAs were significantly different in breast-fed infants and in formula-fed infants. It was observed that the fecal SCFAs were significantly different in both the groups; fecal acetate levels were significantly higher in breast fed infants 
compared to the formula fed infants. Similar findings were observed by Siigur et al where differences in acetate levels were observed between breast fed infants and formula fed infants. There might be different explanations for the latter fact, however, one of the important reasons for this might be the presence of human milk oligosaccharides which is not present in formula milk. It has been reported by Bridgman et al that the formula fed infants had a higher levels of propionates, butyrate and iso-butyrates. Similar findings were reported in clinical trial carried out by Birmingham et al where different levels of valerate and iso-valerate were reported in both the groups. ${ }^{12}$ In addition, similar results were observed in study carried out in Estonia where formula fed infants were reported to have more complex anaerobic flora compared to the mother fed infants. ${ }^{13}$ Higher concentration of iso-butyrates and isovalerate were higher in formula fed babies when compared with breast fed infants in our trial. These findings are in accordance to the findings reported by Mace et al. These results were related to the facts that the proteins present in formula milk might be formed by the metabolism of amino acids present. ${ }^{21}$ The higher absolute levels of SCFAs were seen in formula fed infants and may be related to greater microbial diversity in these infants. This might be one of the reasons for that lead to the metabolism of various substrates present in the colon. Similar results were observed in study carried out in Canada where diverse microbial population in formula fed infants were reported. ${ }^{22}$

\section{CONCLUSION}

It may be concluded that breast milk has different contents compared to the contents of formula milk. Breast feeding is a good practice for infants as breast milk has all the invaluable contents. Findings of this study provide a gateway for direct comparison of gut microbial metabolites of infants' population from Pakistan with European and other geographic population in world. In this study the sampling was done at one time point in early infancy, therefore our results do not give any information on trends in SCFAs over time in perspective to diet.

\section{LIMITATIONS}

In this study the sampling was done at one time point in early infancy, therefore our results do not give any information on trends in SCFAs over time in perspective to diet.

\section{RECOMMENDATIONS}

Follow-up studies must be designed to ascertain how weaning can modify and affect variation in SCFAs production in these infants.

\section{REFERENCES}

1. Byrne CS, Chambers ES, Morrison DJ, Frost $\mathrm{G}$. The role of short chain fatty acids in appetite regulation and energy homeostasis. Int J Obes (Lond). 2015; 39:1331-8.

2. Sa'ad H, Peppelenbosch MP, Roelofsen H, Vonk RJ, Venema K. Biological effects of propionic acid in humans; metabolism, potential applications and underlying mechanisms. Biochimica et Biophysica Acta (BBA)-Molecular and Cell Biology of Lipids. 2010; 1801:1175-83.

3. Puddu A, Sanguineti R, Montecucco F, Viviani GL. Evidence for the gut microbiota short-chain fatty acids as key pathophysiological molecules improving diabetes. Mediators of inflammation. 2014; 2014.

4. Shreiner AB, Kao JY, Young VB. The gut microbiome in health and in disease. Current opinion in gastroenterology. 2015; 31:69.

5. Ríos-Covián D, Ruas-Madiedo P, Margolles A, Gueimonde M, de los Reyes-Gavilán CG, Salazar N. Intestinal short chain fatty acids and their link with diet and human health. Frontiers in microbiology. 2016; 7:185.

6. Arora T, Sharma R, Frost G. Propionate. Anti-obesity and satiety enhancing factor? Appetite. 2011;56:511-5.

7. Hu J, Lin S, Zheng B, Cheung PC. Shortchain fatty acids in control of energy metabolism. Critical reviews in food science and nutrition. 2018; 58:1243-9.

8. Topping DL, Clifton PM. Short-chain fatty acids and human colonic function: roles of resistant starch and nonstarch polysaccharides. Physiological reviews. 2001; 81:1031-64.

9. Kumari M, Kozyrskyj AL. Gut microbial metabolism defines host metabolism: an emerging perspective in obesity and allergic inflammation. Obesity reviews : an official journal of the International Association for the Study of Obesity. 2017; 18:18-31.

10. Azad MB, Konya T, Persaud RR, Guttman DS, Chari RS, Field CJ, et al. Impact of maternal intrapartum antibiotics, method of birth and breastfeeding on gut microbiota during the first year of life: a prospective cohort study. Bjog. 2016; 123:983-93

11. Bridgman SL, Azad MB, Field CJ, Haqq AM Becker AB, Mandhane PJ, et al. Fecal ShortChain Fatty Acid Variations by Breastfeeding Status in Infants at 4 Months: Differences in Relative versus Absolute Concentrations. Frontiers in nutrition. 2017; 4:11-.

12. Edwards CA, Parrett AM, Balmer SE, Wharton BA. Faecal short chain fatty acids in breast-fed and formula-fed babies. Acta paediatrica (Oslo, Norway : 1992). 1994; 83:459-62.

13. Siigur U, Ormisson A, Tamm A. Faecal short-chain fatty acids in breast-fed and bottle-fed infants. Acta paediatrica (Oslo, Norway : 1992). 1993; 82:536-8.

14. Rios-Covian D, Ruas-Madiedo P, Margolles A, Gueimonde M, de Los Reyes-Gavilan CG, Salazar N. Intestinal Short Chain Fatty Acids and their Link with Diet and Human Health. Frontiers in microbiology. 2016; 7:185.

15. Russell WR, Hoyles L, Flint HJ, Dumas ME. Colonic bacterial metabolites and human health. Current opinion in microbiology. 2013; 16:246-54.

16. Balmer SE, Scott PH, Wharton BA. Diet and faecal flora in the newborn: casein and whey proteins. Arch Dis Child. 1989, 64:1678-84.

17. Gil A. Effects of the addition of nucleotides to an adapted milk formula on the microbial pattern of feces in at term newborn infants.
J Clin Nutr Gastroenterol. 1986; 1:127-32.

18. Roberts A, Chierici R, Sawatzki G, Hill M, Volpato S, Vigi V. Supplementation of an adapted formula with bovine lactoferrin: 1 . Effect on the infant faecal flora. Acta Paediatrica. 1992; 81:119-24.

19. Balmer S, Wharton B. Diet and faecal flora in the newborn: iron. Archives of disease in childhood. 1991;66:1390-4.

20. Bridgman SL, Azad MB, Field CJ, Haqq AM, Becker AB, Mandhane PJ, et al. Fecal shortchain fatty acid variations by breastfeeding status in infants at 4 months: differences in relative versus absolute concentrations. Frontiers in nutrition. 2017; 4:11.

21. Macé K, Steenhout $P$, Klassen $P$, Donnet $A$ Protein quality and quantity in cow's milkbased formula for healthy term infants: past, present and future. Protein and Energy Requirements in Infancy and Childhood: Karger Publishers; 2006. p. 189205.

2. Azad MB, Konya T, Persaud RR, Guttman DS, Chari RS, Field CJ, et al. Impact of maternal intrapartum antibiotics, method of birth and breastfeeding on gut microbiota during the first year of life: a prospective cohort study. BJOG: An International Journal of Obstetrics \& Gynaecology. 2016; 123:983-93. 